\title{
Digital Diagnostic Imaging With a Comprehensive PACS: Hypothetical Economic Evaluation at a Large Community Hospital
}

\author{
Rebecca N. Warburton, Paul D. Fisher, Josip Nosil, Gerhard W. Brauer, \\ William J. Lawrence, and Gordon W. Ritchie
}

\begin{abstract}
Since 1983, the 422-bed Victoria General Hospital (VGH) and Siemens Electric Limited have been piloting the implementation of digital medical imaging. including digital acquisition of diagnostic images, in British Columbia. Although full PACS is not yet in place at VGH, experience to date has been used to project annual cost figures (including capital replacement) for a fully digital department. The resulting economic evaluation has been labeled hypothetical to emphasize that some key cost components were estimated rather than observed; this paper presents updated cost figures based on recent revisions to proposed departmental equipment configuration. Compared with conventional diagnostic imaging, digital imaging appears to raise overall annual costs at VGH by nearly $\$ 0.7$ million, (Canadian currency) or $11.6 \%$; this is more favorable than the previous results, which indicated extra annual costs of $\$ 1$ million (16.9\%). Sensitivity analysis still indicates that all reasonable changes in the underlying assumptions result in higher costs for digital imaging than for conventional imaging. Digital imaging appears likely to offer lower radiation exposure to patients, shorter waiting times, and other potential advantages, but as yet the price of obtaining these benefits remains substantial.

(C) 1990 by W.B. Saunders Company.
\end{abstract}

KEY WORDS: medical imaging, picture archiving and communications system, economic costs.

QOME BACKGROUND information is help$\bigcirc$ ful in understanding the work performed at Victoria General Hospital (VGH). Canada has a system of universal medicare that provides all necessary health care services at (in most cases) no cost to the user. The opening of a new hospital in 1984 provided an opportunity to evaluate digital imaging in a community hospital; although VGH receives some regional referrals, it is neither a teaching hospital nor a major tertiary referral center. Currently VGH has a 17 -room medical imaging department with 69.4 full-timeequivalent (FTE) staff, excluding radiologists who are paid on a fee-for-service basis. The department includes facilities for fluoroscopy, general and chest radiography, mammography, computed tomography (CT), angiography, cardiac catheterization, nuclear medicine, and ultrasound, and approximately 65,000 examinations are performed annually. There is some digital acquisition of images and temporary computer image storage, but all permanent image storage remains film-based; the planned final configuration is a filmless department with a picture archiving and communications system (PACS) using predominantly Siemens Electric Ltd (Toronto, Ontario, Canada) equipment.

The original cost projections for digital imaging (1982) indicated that extra capital costs would be more than offset by savings in film costs and film-related staff costs, so that digital imaging would reduce overall annual costs. Potential benefits (reduced radiation, shorter examination times, faster image retrieval, fewer lost images, and teleradiology) merely strengthened the case for conversion to digital. Since then, equipment requirements have increased while examination volumes, film prices, and staff wages have all risen more slowly than expected.

In 1988, senior management of the Ministry of Health requested a revised projection of the final costs for digital imaging at VGH. With the project far from complete, the solution was to perform a hypothetical evaluation using expected costs. The first revision of the 1988 results was presented during $1989^{1}$; this paper is the second revision. More detailed cost modeling and economic evaluation are planned as the project

From the Research and Evaluation Branch, British Columbia Ministry of Health; School of Health Information Science, University of Victoria; and the Department of Medical Imaging, Greater Victoria Hospital Society, Victoria, British Columbia, Canada.

Supported by the British Columbia Ministry of Health and by National Health Research Development Program Grant No. 6610-1437-X.

This research was performed at Victoria General Hospital and the British Columbia Ministry of Health, Victoria, British Columbia, Canada.

Address reprint requests to Rebecca $N$. Warburton, Research and Evaluation Branch, British Columbia Ministry of Health, 1515 Blanshard St, Victoria, BC V8W 3C8, Canada.

(0) 1990 by W.B. Saunders Company.

0897-1889/90/0302-0004\$03.00/0 
proceeds, with the eventual goal of projecting costs for conventional and digital imaging at every hospital in British Columbia. The aim of this paper is to include the full economic costs of conventional and digital imaging at a representative hospital the size of VGH, not simply to catalogue costs for the existing pilot project at VGH. There has been no attempt to model transitional costs because decision makers are interested in the final stage.

It seems clear that digital technology would be preferred to conventional as long as digital imaging costs were equal or lower, making the estimate of relative costs crucial.

\section{MATERIALS AND METHODS}

\section{Main Estimate}

In order to compare medical imaging costs before and after conversion to digital imaging, hypothetical equipment configurations had to be designed for both modes of operation. Capital costs (including replacement cost), maintenance costs, and operating expenses were then projected for each mode. It was assumed that the relative costs of conventional and digital imaging would not be affected by either the number of images ordered or the fees paid to radiologists, hence changes in the mix and volume of procedures have not been modeled and radiologists' fees are not included in the costs shown. Similarly, general hospital overhead costs are not included because it is assumed the value applicable to medical imaging would not be materially affected. The capital value of the building space occupied by both conventional and digital imaging is not included in the capital costs, although annual costs were adjusted for the rental value of space changes.

The conventional imaging configuration was based on the current VGH department; the digital configuration was based on consultations between Siemens and project staff, and is for the most part that described as phase IV in a recently presented paper. ${ }^{2}$ A diagram of the digital department configuration is presented in Fig 1. The goal in designing the digital department configuration was to assemble the minimum-cost system that would deliver services equivalent to a conventional department; the main changes from conventional imaging are the addition of PACS equipment and the elimination of multiformat cameras and filmhandling equipment. This approach was chosen to permit the cost of enhancements (eg, teleradiology) to be compared with their benefits.

Many discussions among all members of the project team were necessary before an "expected" department configuration was agreed on. It must be emphasized that this configuration reflects the combined judgment of the implementation and evaluation team at VGH, not solely the opinion of Siemens. VGH experience with digital acquisition, manipulation, and storage of images was used to build the least-cost system capable of handling the hospital's workload. The expected department configuration includes more computing capacity, more original and backup image storage capacity, and more display stations than in some published PACS configurations; smaller PACS may be incapable of meeting the demands of a working community hospital.

The only additions to conventional department equipment since the 1989 report are a local image network for ultrasound and more computer cabling. The main revisions to the digital department are the addition of PACS interfaces for the radiology information system, nuclear medicine, and ultrasound; and price reductions on some digital acquisition and display equipment. These changes raised the cost of conventional imaging equipment by $\$ 0.3$ million and lowered the cost of digital imaging equipment by $\$ 0.8$ million.

Equipment costs reflect the current price of equipment for a new department; list price minus the normal discount that Siemens applies to such purchases. Special discounts at the pilot site are not reflected in the cost figures.

Capital costs were amortized over a 7-year useful life, discounted at $4 \%$, to produce perpetual annual costs. All costs are in constant 1989 Canadian dollars. General inflation has been excluded from the analysis because it would not affect the relative costs of conventional and digital imaging.

Based on experience at VGH, annual maintenance costs for conventional radiographic equipment were projected at $5.5 \%$ of purchase price. Maintenance for computerized imag. ing equipment was projected at $10 \%$, reflecting the need for software upgrades to keep equipment current for 7 years. For purposes of calculating maintenance costs, all equipment had to be classified as either radiographic or computerized; substantial computerized equipment is used in a conventional imaging department, particularly in cardiac catheterization, angiography, CT, nuclear medicine, and ultrasound.

Staffing costs for conventional imaging were based on VGH costs, with adjustments to make costs more typical where necessary (eg, partial year costs for cardiac catheterization were amended to full year costs). Staff savings after conversion to digital imaging were projected by interviews with department staff to determine the amount of time spent on film-related tasks (15.8 FTEs), with some confirmation by time-and-motion studies. Management of the PACS was assumed to require a system manager, a programmer, and a round-the-clock computer operators (total, 4.4 FTEs). Figure 2 shows the staffing changes within medical imaging.

Supply costs were estimated by assuming that only $10 \%$ of current film-related supplies would be required after conversion, primarily to handle patient transfers; computer supplies were added for the digital department.

Adjustments were also made to reflect the true economic cost of significant nonbudget items, including the value of space changes and silver recovery. Improved productivity is expected to permit the digital department to use one less examination room than conventional, and the 1-year and 5-year film archives would of course be replaced with a computer image archive. Market-equivalent rents for space occupied by the examination room, film archives, film processors, and multiviewers were added to conventional department annual costs (\$10.50 per square foot in the department, $\$ 5.25$ per square foot for the offsite 5-year archive). Silver recovery revenue (normally included with general hospital revenues) was included as conventional department annual revenue, and the silver recovery value of films in the 1 -year 


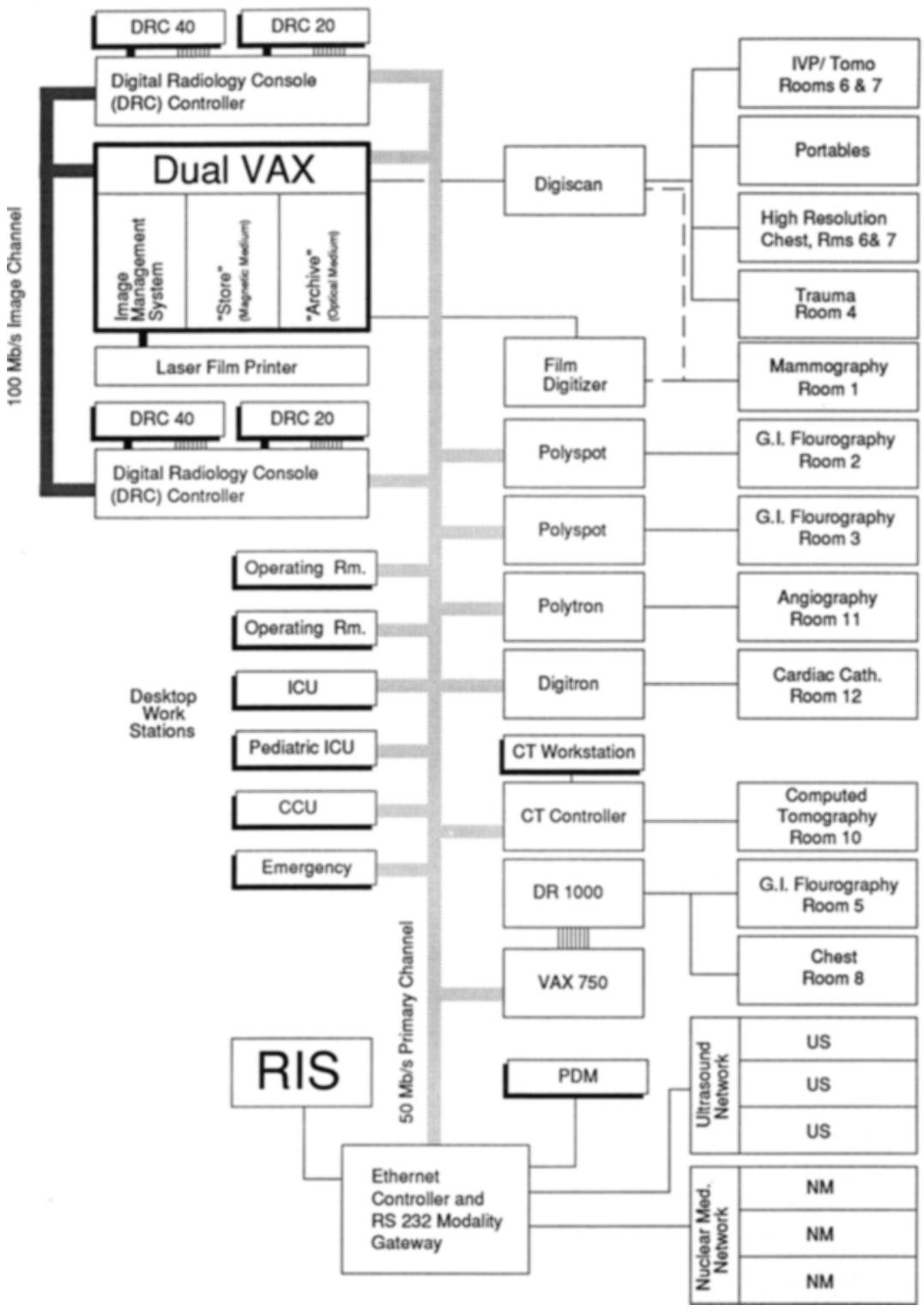

ital department configuration.

and 5-year archives was added to conventional department capital costs. Finally, the market-equivalent rent for additional computer space was added to digital department annual costs, and the cost of site preparation for computer rooms was added to digital department capital costs.

Once complete, annual capital and operating costs were combined into a single figure, yielding real (no inflation) annual economic (not accounting) costs in perpetuity. Figure 3 shows the changes in major cost categories. More detail appears in an appendix available from the authors.

\section{Sensitivity Analysis}

Sensitivity analysis was performed to test the resilience of the conclusions under a variety of different conditions; the analysis was informed by noting areas of uncertainty in the cost estimates. In general, the conclusions of economic evaluation studies are greatly strengthened if no plausible changes in the underlying assumptions alter the direction of the results. Sensitivity analysis is particularly important for hypothetical studies incorporating estimates rather than observations.

In all, seven basic scenarios were calculated: expected, high equipment costs (plus $10 \%$ to $11 \%$ ), low equipment costs (minus $10 \%$ to $13 \%$ ), high discount rate $(6 \%)$, low discount rate $(2 \%)$, short amortization period ( 5 years), long amortization period (10 years).

From these basic scenarios, three further scenarios were derived: overall worst case, overall best case, and "break even." The worst case uses high costs for digital imaging and expected costs for conventional, except that for equipment common to digital and conventional departments, consistent 


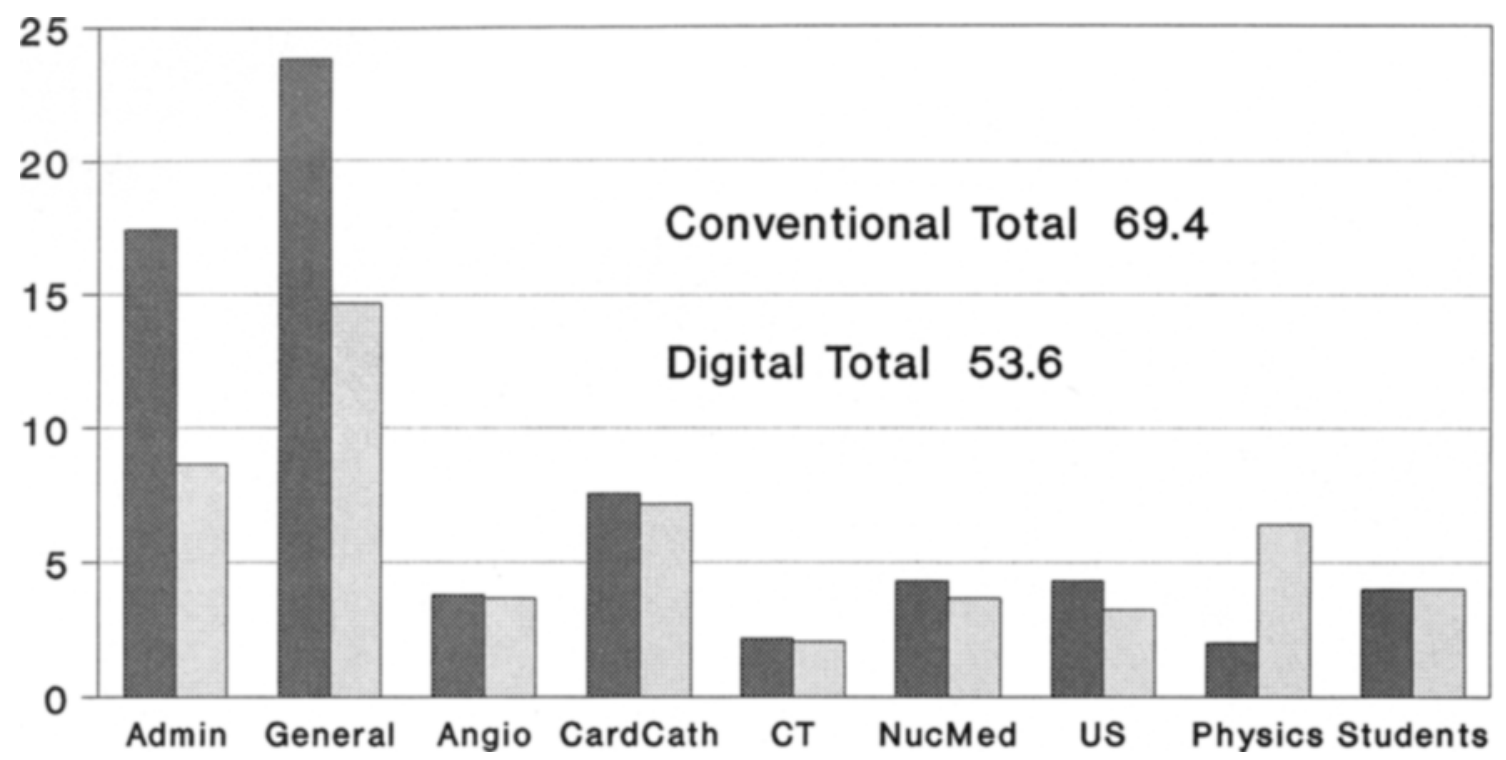

Fig 2. Expected full-time-equivalent staff in medical imaging, excluding radiologists. Dark bar, conventional; light bar, digital.

cost assumptions must be used; the worst case uses either high or low equipment costs, depending on which yields the worse result for digital, in calculating costs for both digital and conventional departments.

Similarly, the best case uses low costs for digital and expected costs for conventional, with comparable adjustments to ensure consistency for equipment common to digital and conventional departments.
The break-even scenario works backwards from total digital imaging costs equal to expected total conventional costs in order to calculate the resulting value of capital equipment.

The results of the sensitivity analysis are summarized in Figs 4 and 5. All scenarios are intended to represent the limits for plausible values, not extreme or impossible values. Even the best and worst case scenarios do not combine all possible

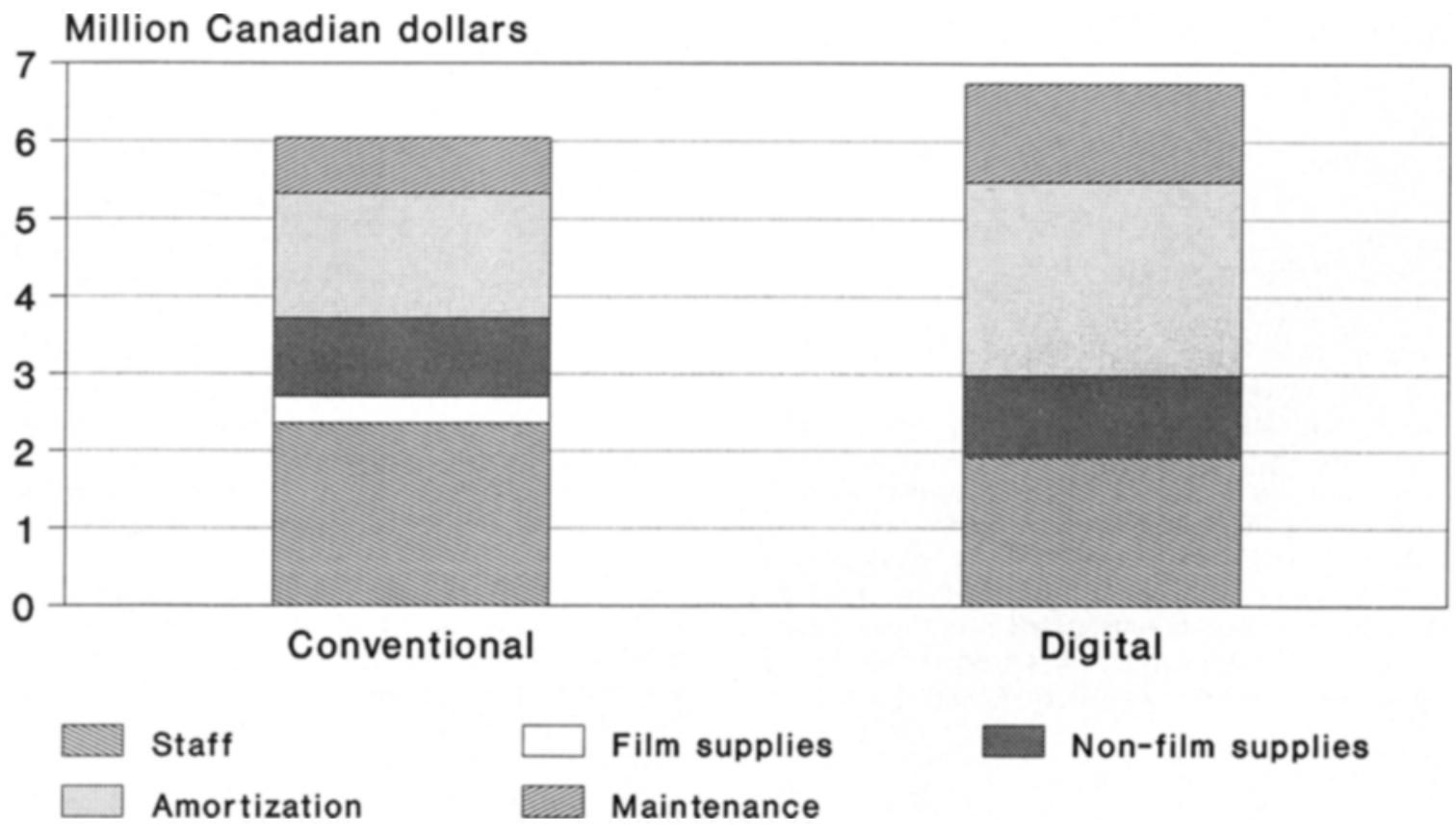

Fig 3. Expocted annual costs for medical imaging. 


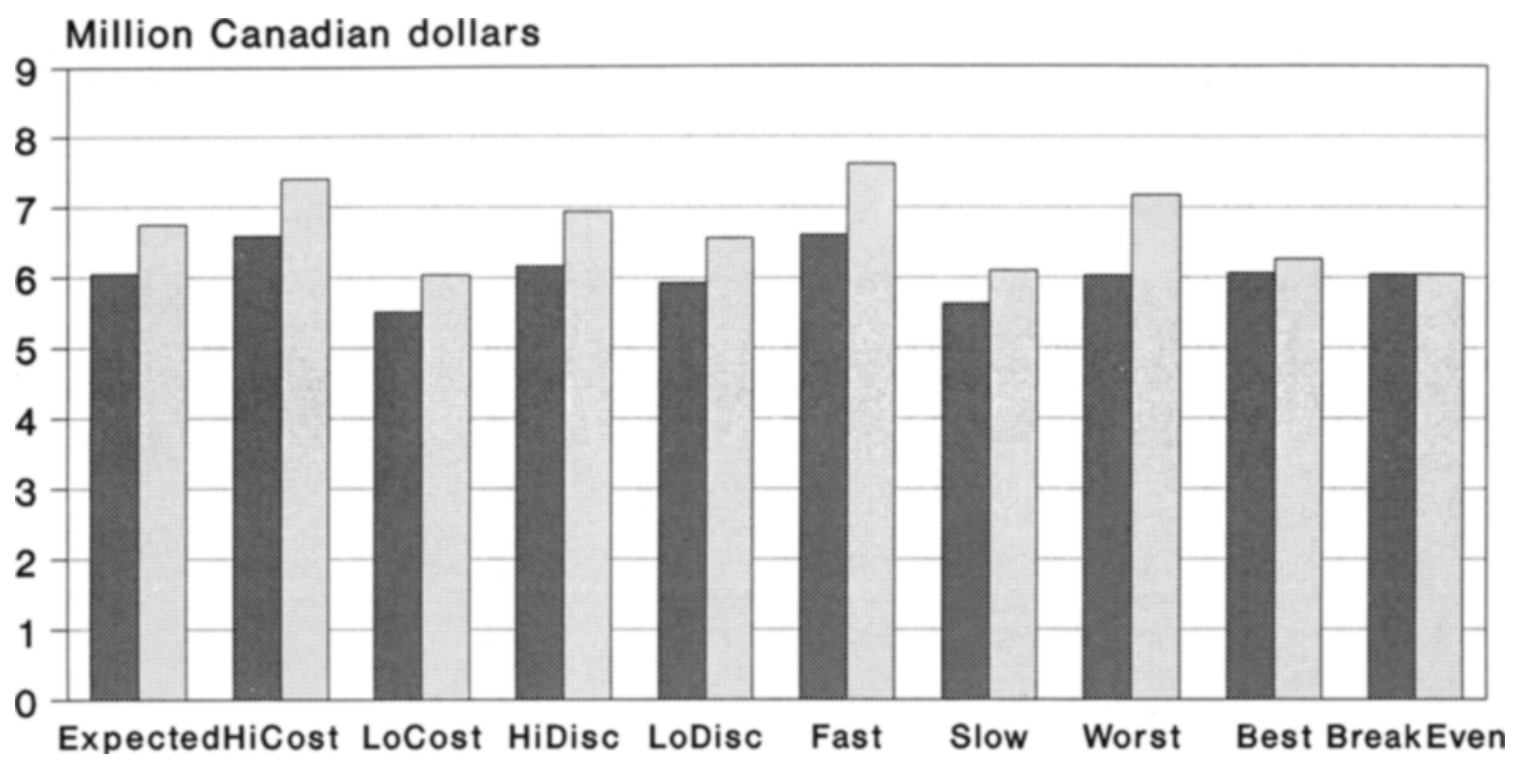

Fig 4. Estimated annual costs for medical imaging in various scenarios. HiCost, high equipment costs; LoCost, low equipment costs; HiDisc, high discount rate; LoDisc, low discount rate; Fast, short amortization period; Slow, long amortization period. Dark bar, conventional; light bar, digital.

positive or negative values in one scenario, because this occurrence was considered highly unlikely.

\section{RESULTS}

The analysis shows that in no case did the savings from elimination of film offset the extra capital costs required for digital imaging. Over- all, digital imaging costs significantly more than conventional imaging, ranging from $\$ 0.2$ million (3.4\%) more for the best case to $\$ 1.1$ million (18.9\%) for the worst case; the expected scenario shows extra costs of $\$ 0.7$ million (11.6\%). The break-even scenario shows maximum capital costs of \$12.4 million for digital imaging, \$2.6 million

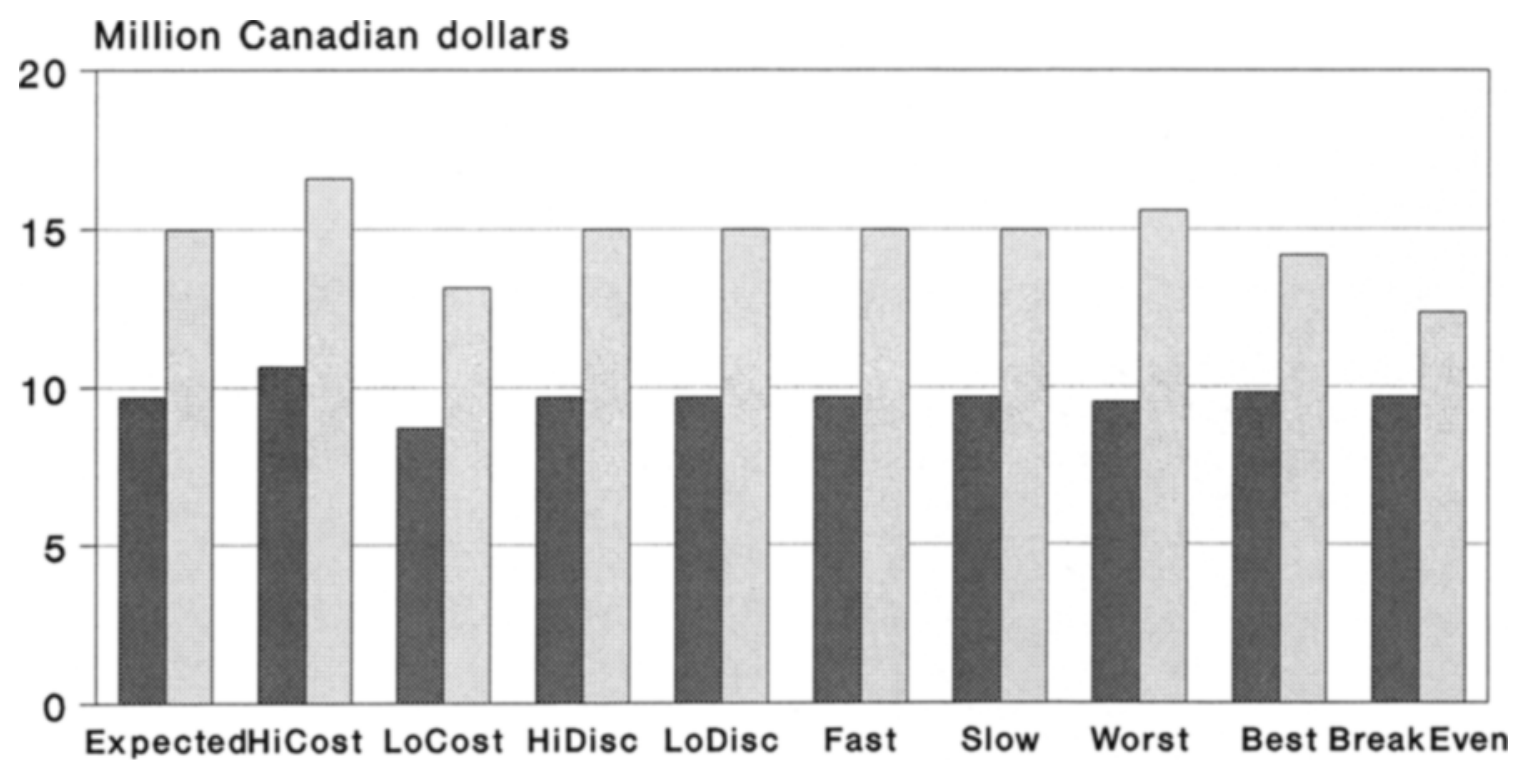

Fig 5. Expected capital costs for medical imaging. HiCost, high equipment costs; LoCost, low equipment costs; HiDisc, high discount rate; LoDisc, low discount rate; Fast, short amortization period; Slow, long amortization period. Dark bar, conventional; light bar, digital. 
less than expected costs and $\$ 0.8$ million less than even the low-cost scenario. The expected value of conventional department equipment is $\$ 9.7$ million.

These results are more favorable than the previous results, which indicated expected extra costs of $\$ 1.0$ million (16.9\%) and ranged from $\$ 0.5$ million $(7.8 \%)$ for the best case to $\$ 1.5$ million $(25.6 \%)$ for the worst case.

\section{DISCUSSION}

This finding is consistent with some reported cost studies of digital imaging, ${ }^{3,4}$ and inconsistent with others. ${ }^{5,6}$ As a recent survey paper indicates, ${ }^{7}$ there are many reasons for the different results; equipment configurations, prices, and maintenance costs vary significantly, amortization methods differ, and there are wide differences in the estimates of staff and supply savings. Because the sensitivity analysis showed consistent results despite wide variations in the relevant variables, we are confident that digital imaging installed in a similar configuration at any similar hospital in 1989 would be more costly than an equivalent conventional diagnostic imaging department, regardless of the vendor.

However, broadening the scope from the medical imaging department to the entire hospital could produce a different result. A 1986 survey of US radiologists ${ }^{8}$ and a 1987 survey of US hospital administrators ${ }^{9}$ showed that these professionals believed digital imaging with a PACS would shorten the average length of stay by $13 \%$ and $6 \%$, respectively, but no data to substantiate these opinions has been produced by any center investigating PACS. In the US health care system, such improved productivity would result in higher hospital revenues without any added costs for direct patient care. For British Columbia hospitals, funded via global budgets, higher productivity would not affect revenues directly. Nonetheless, reducing stays has value in any system; shorter stays could permit bed closures or shorten waiting lists for elective procedures.

Greater Victoria Hospital Society statistics unrelated to the digital imaging project ${ }^{10}$ show that $1 \%$ of patients whose care was reviewed by a utilization management team had their stays lengthened at some point by delays in medical imaging. The magnitude and cause of the observed delays was not recorded, making the extent to which conversion to digital imaging would prevent such delays unclear. However, based on a brief analysis of costs at VGH, it appears that closing acute beds would result in cost savings of $\$ 270$ to $\$ 360$ per day. (The average total cost per day is approximately $\$ 450$.) In the expected cost scenario, reducing the average 6.5-day acute stay to 6.4 days would permit sufficient bed closures to offset the projected extra costs of digital imaging. This reduction (less than $2 \%$ ) appears possible with conversion to digital imaging, and work now in progress at VGH is investigating the probable effects of conversion to digital imaging on medical imaging delays and hospital stays.

Estimating the value of the saved bed-days, left in service, is much more complex and has not been attempted here. In theory, the value of shortening a waiting list is related to the difference in health status (or quality of life) of patients before, during, and after admission for treatment; shorter waiting lists would not alter the effects of treatment but would reduce the time before treatment, hastening the resulting change in health status. The value of patients' work and leisure time, and close family members' time, are important. Because waiting lists are frequently affected by constraints in operating room staffing or equipment availability, freeing hospital beds would not affect all waiting lists equally. If, as seems likely, shorter stays meant that hospital inpatients would, on average, have worse health status than when stays were longer (increased acuity), staffing might need to be increased and average operating costs per bed might increase. In any event, because of the subjectivity involved in the required estimates of the value of time, the final result would not permit an objective determination of the reduction in hospital stay that digital imaging would need to produce in order to break even.

\section{CONCLUSION}

Continued rapid technological progress is likely to lower the cost of digital imaging relative to conventional, and to improve the performance of digital relative to conventional. Although digital imaging is currently more costly than conventional imaging, it appears likely that over the next 2 to 5 years the potential benefits of digital imaging will be realized and equivalence between 
conventional and digital imaging costs will be demonstrated. Because clinical and other benefits appear to make digital imaging preferable to conventional imaging once costs are equivalent, digital technology should not be ignored in the planning of medical imaging departments scheduled to open in 1992 or beyond.

\section{ACKNOWLEDGMENT}

The authors would like to acknowledge the invaluable assistance of the staff of the Medical Imaging Department of the Greater Victoria Hospital Society, and in particular Robert P. Clark and Barbara A. Hofner, in the preparation of cost estimates; and thank the Greater Victoria Hospital Society and Ministry of Health staff who assisted with this study.

\section{REFERENCES}

1. Parrish D, Warburton RN, Zelman W: Cost and economic analysis for PACS: A tutorial. Proceedings of Image Management and Communications, Washington, DC, 1989, pp 262-269

2. Fisher PD, Brauer GW, Nosil J, et al: Comprehensive computerized medical imaging (CCMI) at Victoria General Hospital: Final implementation plan. SPIE Med Imaging IV, 1990 (in press)

3. Saarinen AO, Haynor DR, Loop JW, et al: Modelling the economics of PACS: What is important? SPIE Med Imaging III 62-73:1989

4. Andriessen JHTH, ter Haar Romeny BM, et al: Savings and costs of a picture archiving and communication system in the University Hospital Utrecht. SPIE Med Imaging III 578-584:1989

5. Arenson RL, Seshadri SB, Hiss S, et al: PACS at Penn. SPIE Med Imaging III 50-59:1989
6. Cywinski JK, Vanden Brink JA: Review of experience with PACS cost analysis model. SPIE Med Imaging III 535-538:1989

7. van Gennip EMSJ, Ottes FP, van Poppel BM, et al: Why do cost-benefit studies of PACS disagree? SPIE Med Imaging IV, 1990 (in press)

8. Vanden Brink JA: Medical Image Processing, Archiving, and Communication (PACS) Tracking Study. Proprietary study by Technology Marketing Group, Des Plaines, IL, 1986

9. Vanden Brink JA: Medical Image Processing, Archiving, and Communication (PACS) Tracking Study. Proprietary study by Technology Marketing Group, Des Plaines, Illinois, 1987

10. Quality and Utilization Management Program of the Greater Victoria Hospital Society: Internal data, 1989 